\title{
AVALIAÇÃO DE IDEIAS EM INICIATIVAS CROWDSOURCING PARA INOVAÇÃO: UM ESTUDO EXPLORATÓRIO
}

\author{
Joade Cortez Gomes (joadecgomes@yahoo.com.br) - UFRN \\ Mario Orestes Aguirre González (mario@ct.ufrn.br) - UFRN \\ Marcela Squires Galvão Fernandes (marcela@ reitoria.ufrn.br) - UFRN \\ Lucas Matheus Rodrigues (lucasmtrd@gmail.com) - UFRN \\ Janine Kharen de Lima Ferreira (janinekharen@hotmail.com) - UFRN \\ Bruno Colt Ferreira do Nascimento (brunocolt.ufrn@gmail.com) - UFRN \\ Técia de Lima Silva (tecia-lima@ @otmail.com) - UFRN \\ Rafael Monteiro de Vasconcelos (rafaelmonteirov@yahoo.com.br) - UFRN
}

\begin{abstract}
RESUMO
O processo de crowdsourcing parte de um tema ou problema a ser resolvido pela organização, aberto à multidão que interage e participa do processo resolutivo por meio de ideias, submetendo-se ao final a um processo avaliativo no qual as melhores são selecionadas e implementadas. $O$ desafio é a assertividade da seleção, principalmente quando há um alto volume de contribuições. O objetivo deste artigo é propor elementos para avaliação de ideias geradas a partir de uma abordagem crowdsourcing. Para isso, realizou-se uma revisão bibliográfica sistemática e, por meio de um estudo de caso, validou-se no Escritório de Ideias e Inovação da Universidade Federal do Rio Grande do Norte os elementos de avaliação de ideias: Filtro popular e técnico; Detalhamento das ideias; Avaliação final por multicritérios e análise consensual; e Fatores influenciadores..
\end{abstract}

Palavras-chave:Escritório de Ideias; gerenciamento de ideias; criatividade coletiva; crowdsourcing; open innovation

Área: Potencial da Internet of Things (IoT) para o desenvolvimento de produtos e serviços

\section{INTRODUÇÃO}

As organizações públicas necessitam estar adaptadas para responder com eficiência e qualidade aos usuários e, para isso, precisam estar comprometidas com modernas práticas de gestão. Promover a criatividade no ambiente público e gerar propostas inovadoras para este requer uma abertura a novas ideias e o apoio a mudanças por parte da organização. A pesquisa de Soares (2009) relata alguns caminhos para a promoção de um ambiente de inovação no serviço público, como: canais de comunicação eficientes em todos os níveis da organização; tarefas desafiantes ao potencial criador; normas mais flexíveis; descentralização do poder; valorização da iniciativa e da participação na tomada de decisão; política de benefícios e sistema de recompensa voltada às ideias inovadoras; estímulo da chefia para novas ideias; respeito às opiniões divergentes; e capacitação dos agentes públicos para o desenvolvimento de seu potencial criador. 
Nos últimos anos, uma abordagem colaborativa que vem ganhando muita atenção no mundo é o crowdsourcing (CHIU; LIANG; TURBAN, 2014), podendo ser visto como um modelo para atrair um público interessado, motivado e capaz de fornecer soluções superiores em qualidade e quantidade para aqueles que podem mesmo nas formas tradicionais de colaboração (BRABHAM, 2008). O processo de crowdsourcing pode assumir vários formatos e composições, como por exemplo, open innovation, co-creation, collective intelligence, user innovation e open source, mas a sua estrutura parte de um tema ou problema a ser resolvido pela organização, onde a tarefa é submetida pela rede à multidão, que interage e participa do processo resolutivo por meio de ideias, submetendo-se ao final a um processo avaliativo no qual as melhores são selecionadas e implantadas (CHIU; LIANG; TURBAN, 2014). Há três principais elementos associados ao processo de resolução criativa: identificação de problemas, geração de ideias e avaliação de ideias (REITER-PALMON; ILLIES, 2004).

Nessa direção, alinhada a políticas de desenvolvimento e inovação, a Universidade Federal do Rio Grande do Norte (UFRN) prevê em sua missão e em suas diretrizes estratégicas a inovação como um fator norteador para as suas ações. Assim, no sentido de uma gestão participativa, a Universidade vem trabalhando desde 2014 o Escritório de Ideias e Inovação, que atua essencialmente nos processos de captação, avaliação e implantação de projetos na organização.

Ao utilizar o crowdsourcing como processo de atuação, o Escritório de Ideias e Inovação busca a solução de problemas de forma integrativa com a comunidade universitária (servidores e alunos), estimulando a mudança de cultura, direcionando-a para a utilização da criatividade. Além disso, há com essa abordagem, o empoderamento dos envolvidos e a elevação da motivação intrínseca da comunidade.

Como o crowdsourcing desencadeia um cenário de grande quantidade de ideias geradas, um dos desafios que se apresenta nesse processo é a assertividade na seleção das ideias a serem desenvolvidas, assim o objetivo do artigo é propor elementos para avaliação de ideias geradas a partir de uma abordagem crowdsourcing.

O artigo está estruturado em 5 seções, com a primeira parte introdutória. A seção 2 contempla o método da pesquisa, seguido do referencial teórico. A sessão 4 apresenta o estudo de caso, com a descrição dos elementos para avaliação de ideias. Por fim, a sessão 5 traz as considerações finais, limitações e recomendações para futuras pesquisas.

\section{MÉTODO DE PESQUISA}

O artigo pode ser classificado como uma pesquisa descritiva (GIL, 2009), com abordagem qualitativa (CRESWELL, 2010), cujo procedimento técnico foi um estudo de caso (YIN, 2010). O procedimento da pesquisa encontra-se descrito na Figura 1.

Figura 1. Resumo do procedimento da pesquisa. Fonte: elaboração própria

\begin{tabular}{|c|c|c|}
\hline Fundamentação teórica & $\begin{array}{l}\text { Análise e obtenção dos } \\
\text { elementos para avaliação }\end{array}$ & $\begin{array}{c}\text { Validação dos elementos de } \\
\text { avaliação }\end{array}$ \\
\hline $\begin{array}{ll}\text { - } & \text { Identificação do } \\
\text { estado da arte } \\
\text { - } \\
\text { Elaboração do modelo } \\
\text { conceitual }\end{array}$ & 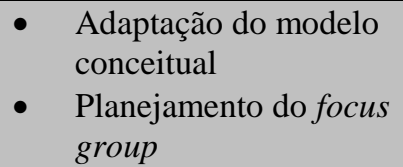 & 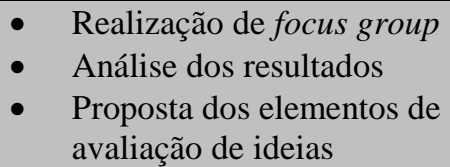 \\
\hline
\end{tabular}

A fundamentação teórica realizada via pesquisa bibliográfica sistemática focou nos temas crowdsourcing e avaliação de ideias e contou com a leitura de 83 artigos, além de alguns livros, dissertações e teses, compilando as informações em uma planilha. Os questionamentos 
que nortearam a pesquisa foram: Qual o conceito de crowdsourcing? Quais são as suas tipologias? Como estrutura-se o seu funcionamento? Quais os critérios que são utilizados num processo de avaliação de ideias? Quais os métodos utilizados? Quais os fatores que influenciam nesse processo?

De posse do estado da arte, foram levantados elementos para a avaliação de ideias. Adaptando-se de acordo com as características do processo de inovação e melhorias da UFRN, os elementos foram postos à validação em uma sessão de focus group. Na sessão, as informações geradas foram resultados da contribuição de uma equipe multidisciplinar de relevância no contexto da UFRN: administração, gestão de pessoas, criatividade e inovação, gestão de projetos, eficiência energética, engenharia, tecnologia da informação, programação web e design.

Com a análise dos resultados da sessão do focus group, estruturou-se uma proposta dos elementos que compõem um sistema de avaliação de ideias geradas a partir de uma abordagem crowdsourcing.

\section{GERAÇÃO E AVALIAÇÃO DE IDEIAS}

\subsection{Crowdsourcing}

Motivadas pelas constantes mudanças ocorridas na sociedade como um todo, as organizações buscam em seus servidores perfis criativos para lidar com a pressão e a dinamicidade do contexto. Novas oportunidades surgem, novos produtos e serviços são desenvolvidos e novos modelos e ferramentas de negócio estão sendo empregados, como é o caso do crowdsourcing.

Em um ambiente global cada vez mais tecnológico, o crowdsourcing deriva de uma tendência geral onde a tecnologia da informação permite que ideias e esforços possam ser compartilhados abertamente por meio da Internet. Para Brabham (2013), crowdsourcing pode ser entendido como uma resolução de problemas e produção de modelos distribuídos em rede online, que aproveita as redes para reunir informações e distribuir as tarefas de grande escala ou solicitar ideias para os problemas existentes como um desafio.

Há na literatura alguns estudos que buscam classificar o crowdsourcing principalmente quanto aos critérios motivadores. Howe (2008) orienta-se pelo papel estratégico do modelo, Geiger et al. (2012) classificam o processo a partir da visão do sistema de informação, já Saxton et al. (2013) utilizam o processo de gestão e os níveis de controle adotados nas iniciativas, conforme pode ser observado no Quadro 1.

Quadro 1. Classificação por critérios do crowdsourcing. Fonte: elaboração própria

\begin{tabular}{|c|c|c|}
\hline Autor & Critério & Classificação \\
\hline Howe (2008) & $\begin{array}{l}\text { Papel estratégico do } \\
\text { modelo }\end{array}$ & $\begin{array}{c}\text { Crowd Wisdom ou Collective intelligence } \\
\text { Crowd Voting } \\
\text { Crowd Creation } \\
\text { Crowd Funding }\end{array}$ \\
\hline Saxton et al. (2013) & Sistema de informação & $\begin{array}{c}\text { Modelo intermediário } \\
\text { Modelo de produção mídia cidadã } \\
\text { Modelo de desenvolvimento de software } \\
\text { colaborativo } \\
\text { Modelo de vendas de produtos digitais } \\
\text { Modelo de produto de design } \\
\text { Modelo de financiamento social } \\
\text { Modelo de consumidor relator } \\
\text { Modelo de construção de base do } \\
\text { conhecimento } \\
\text { Modelo de projetos de ciência colaborativa }\end{array}$ \\
\hline
\end{tabular}




\begin{tabular}{|c|c|c|}
\hline & & Crowd processing \\
Geiger et al. (2012) & Processo de gestão e os & Crowd rating \\
& níveis de controle & Crowd solving \\
& & Crowd creation \\
\hline
\end{tabular}

Assim, qualquer organização que visa à adoção do crowdsourcing de uma forma eficaz, é necessário considerar cuidadosamente as características do processo que será utilizado para atingir seus objetivos em particular.

Para este artigo, adotou-se o modelo tipológico de Howe (2008) como referência, sendo aplicados os tipos Crowd Wisdom e Crowd Voting. O primeiro utiliza a sabedoria e inteligência coletiva para resolver problemas e fornecer insights e ideias, gerando inovação de produtos, processos ou serviços. Já o segundo aproveita a multidão para avaliar e dar opiniões sobre as ideias geradas por meio de voto.

\subsection{Avaliação de Ideias}

De acordo com Reiter-Palmon e Illies (2004), há três principais processos associados com o processo de solução criativa de problemas: identificação de problemas, geração de ideias, e avaliação ideias. O processo de avaliação sistemática de ideias em sua fase inicial é de suma importância para as organizações (BLOHM et al., 2016) e o sucesso da implementação de um projeto é bastante influenciado pelo processo de avaliação das ideias.

No processo de inovação, os custos associados em avaliar erroneamente uma ideia má como boa (erro tipo I) podem ser significativamente diferentes dos custos de avaliar uma boa ideia como ruim (erro tipo II). Implementar ideias no primeiro caso, refletirá em uma má alocação de recursos financeiros da organização, enquanto o último caso pode refletir em uma perda de oportunidade, podendo ser fatal para a organização. O risco de ocorrência e consequentes erros de classificação geralmente aumentam com a concentração de ideia de qualidade em um pequeno número de ideias muito boas (CAROFF; BESANÇON, 2008). No entanto, os resultados da pesquisa dos autores mostram que a qualidade da decisão de leigos aumenta em tais condições. Além disso, o erro tipo II pode ser minimizado quando as comunidades de inovação online funcionam como pontos de discussões intensas de seus membros ou quando a organização utiliza essas discussões qualitativas em consideração no processo de avaliação.

Quase todos os métodos de avaliação de ideias utilizam como base a aplicação de critérios por juízes (GABRIEL et al., 2016). Partindo do pressuposto que criatividade é algo novo, Dean et al. (2006) esclarecem que uma ideia criativa pode ser avaliada pelo conceito de multicritério (Quadro 2). Na literatura, é encontrada a aplicação de critérios de avaliação de ideias por diversos autores em seus estudos, no entanto, poucos justificam a utilização do critério utilizado. O Quadro 3 apresenta um resumo dos critérios encontrados e seus respectivos autores.

Quadro 2. Critérios para medição da qualidade das ideias. Fonte: adaptado de Dean et al. (2006)

\begin{tabular}{|c|c|c|c|}
\hline Novidade & Viabilidade & Relevância & Especificidade \\
\hline $\begin{array}{c}\text { Originalidade } \\
\text { Paradigma }\end{array}$ & Aceitabilidade & Aplicabilidade & Explicitação \\
& Implementabilidade & Efetividade & Plenitude \\
& & & Clareza \\
\hline
\end{tabular}

Quadro 3. Critérios aplicados a avaliação de ideias. Fonte: elaboração própria

\begin{tabular}{|c|c|}
\hline Critérios & Autor \\
\hline Novidade, viabilidade, relevância, especificidade & Dean et al. (2006) \\
\hline
\end{tabular}




\begin{tabular}{|c|c|}
\hline Originalidade, viabilidade & Rietzschel et al. (2006) \\
\hline Impacto estratégico, oportunidade financeira, benefícios, riscos & Kahraman et al. (2007) \\
\hline $\begin{array}{c}\text { Criatividade, Potencial do Mercado, Qualidade, Demanda de } \\
\text { Negócios, Estratégia }\end{array}$ & Ebner et al. (2009) \\
\hline Customização, originalidade, viabilidade & Tung et al. (2009) \\
\hline Novidade, utilidade, viabilidade & Gray et al. (2010) \\
\hline Oportunidade financeira, satisfação do consumidor, viabilidade & Xie e Zhang (2010) \\
\hline Qualidade, originalidade & $\begin{array}{l}\text { Herman e Reiter-Palmon } \\
\text { (2011) }\end{array}$ \\
\hline $\begin{array}{l}\text { Novidade, utilidade, viabilidade, nível de detalhe, forma de } \\
\text { apresentação }\end{array}$ & Liikkanen et al. (2011) \\
\hline Criatividade, novidade, utilidade, viabilidade & $\begin{array}{l}\text { Kudrowitz e Wallace } \\
(2013)\end{array}$ \\
\hline Quantidade, variedade, novidade, qualidade & Verhaegen et al. (2012) \\
\hline Produtibilidade, originalidade, valor agregado & Magnusson et al. (2014) \\
\hline $\begin{array}{c}\text { Alinhamento estratégico, viabilidade, retorno financeiro, social e } \\
\text { ambiental }\end{array}$ & Correa e Ferreira (2015) \\
\hline Velocidade, qualidade & Eling et al. (2015) \\
\hline Qualidade, novidade e variedade & Jagpat et al. (2015) \\
\hline Novidade, variedade, quantidade, qualidade & Cluzel et al. (2016) \\
\hline $\begin{array}{l}\text { Riscos, valor agregado, sustentabilidade, qualidade da apresentação, } \\
\text { dificuldade de implementação, originalidade }\end{array}$ & Gabriel et al. (2016) \\
\hline Originalidade & Hao et al. (2016) \\
\hline
\end{tabular}

Blohm et al. $(2011$; 2016) destacam que uma escala multicritérios é superior para se avaliar ideias de base comunitária em termos de precisão na avaliação e satisfação dos envolvidos. Riedl et al. (2013) corroboram e acrescentam que a aplicação da escala de avaliação multicritério leva a uma maior qualidade da decisão em comparação com a escala de classificação com critérios únicos.

Auxiliando a tomada de decisão no processo avaliativo, métricas e indicadores são utilizados para avaliar o desempenho das ideias. Dentre as encontradas na literatura, a Análise Consensual considera uma iniciativa como criativa quando há concordância entre os avaliadores, que trabalham de forma independente e são guiados por concepções subjetivas individuais sobre a criatividade (HENNESSEY; AMABILE, 2011). Um valor de concordância é aceitável quando atinge o nível de 0,70 ou mais e, quando alcança esse patamar, é apropriado calcular uma soma (ou uma média) em todas as classificações atribuídas a cada iniciativa.

Considerando que o ato de avaliar uma ideia é resultado da percepção do avaliador, seja um especialista ou mesmo a multidão, pode-se atribuir esse processo como um momento de tomada de decisão, onde um conjunto de fatores (Quadro 4), intrínsecos e extrínsecos, influencia a qualidade do seu resultado.

Quadro 4. Fatores que influenciam o processo de avaliação de ideias. Fonte: elaboração própria

\begin{tabular}{|c|c|c|}
\hline Fatores Influenciadores & Descrição & Autores \\
\hline $\begin{array}{c}\text { Ambiguidade das ideias } \\
\text { (Sensemaking) }\end{array}$ & $\begin{array}{c}\text { Grau de efetivo entendimento das ideias } \\
\text { pelos avaliadores. }\end{array}$ & Gutiérrez (2014) \\
\hline Apresentação das ideias & $\begin{array}{c}\text { Eficiência, atratividade e acessibilidade do } \\
\text { canal de apresentação. }\end{array}$ & $\begin{array}{c}\text { Onarheim } \text { et al. (2012); } \\
\text { Magnusson } \text { et al. (2014) }\end{array}$ \\
\hline
\end{tabular}




\begin{tabular}{|c|c|c|}
\hline Clareza do problema & $\begin{array}{c}\text { O grau de entendimento do que pretende-se } \\
\text { solucionar }\end{array}$ & Kobayashi et al. (2009) \\
\hline Condição de trabalho & $\begin{array}{c}\text { Sobrecarga de trabalho (baixa } \\
\text { disponibilidade de tempo, alta carga de } \\
\text { pressão e estresse) }\end{array}$ & $\begin{array}{l}\text { Blair e Mumford (2007); } \\
\text { Eubanks e Mumford } \\
\text { (2010) }\end{array}$ \\
\hline $\begin{array}{l}\text { Conhecimento da } \\
\text { Organização }\end{array}$ & $\begin{array}{l}\text { Grau de conhecimento da organização e } \\
\text { dos objetivos desejados }\end{array}$ & $\begin{array}{l}\text { Eubanks e Mumford } \\
\text { (2010) }\end{array}$ \\
\hline $\begin{array}{l}\text { Contexto histórico e } \\
\text { cultural }\end{array}$ & $\begin{array}{c}\text { Interferência histórica e culturais presentes } \\
\text { na sociedade }\end{array}$ & Csikszentmihalyi (1999) \\
\hline Entendimento dos critérios & $\begin{array}{c}\text { Capacidade de aplicação correta e } \\
\text { uniforme dos critérios }\end{array}$ & $\begin{array}{c}\text { Dean } \text { et al. (2006); } \\
\text { Verhaegen } \text { et al. (2012) }\end{array}$ \\
\hline Motivação & $\begin{array}{l}\text { Motivação intrínseca do indivíduo. O } \\
\text { quanto participar do processo faz sentido } \\
\text { para os envolvidos. }\end{array}$ & Amabile (2012) \\
\hline Ownership & Senso de propriedade sob a ideia & Onarheim et al. (2012) \\
\hline Perfil dos Avaliadores & Perfil de criatividade. Foco regulador. & $\begin{array}{c}\text { Von Oech (1994); } \\
\text { Csikszentmihalyi (2001); } \\
\text { Herman e Reiter-Palmon } \\
\text { (2011) }\end{array}$ \\
\hline Quantidade de ideias & $\begin{array}{c}\text { Disponibilidade de tempo para } \\
\text { envolvimento em grande número de ideias }\end{array}$ & Yang (2012) \\
\hline
\end{tabular}

Para a aplicação da pesquisa utilizou-se como critério de avaliação, aqueles propostos por Dean et al. (2006): novidade, viabilidade, relevância e especificidade. O critério novidade é visto como uma questão chave para se medir a criatividade das ideias, uma vez que ela mensura o grau em que uma ideia é original e modifica um paradigma existente; a viabilidade pode ser entendida pelo grau em que a ideia é aceitável e implementável, ou seja, se as restrições são conhecidas e se há viabilidade técnica; uma ideia é relevante se for aplicada especificamente para o problema e se resolvê-lo efetivamente; por fim, o critério especificidade está relacionado com a explicitação, plenitude e clareza da ideia, ou seja, se ela é comunicável.

Dessa forma, considerando o (1) tipo de crowdsourcing, (2) os critérios de avaliação, (3) os fatores que influenciam o processo de avaliação de ideias e (4) as características da UFRN e do modelo já utilizado pelo Escritório de Ideias e Inovação, o artigo utilizou os seguintes elementos: filtro popular e técnico; detalhamento de ideias; avaliação final por multicritérios e análise consensual; e os fatores influenciadores apresentados no Quadro 4.

\section{ESTUDO DE CASO}

\subsection{UFRN e Escritório de Ideias e Inovação}

De acordo com a visão estratégica da UFRN, prevista no Plano de Desenvolvimento Institucional (PDI) 2010-2019, a universidade deve buscar inovação. Na mesma linha, de acordo com Plano de Gestão 2015-2019, a instituição deve disseminar a cultura da inovação tecnológica e suas repercussões enquanto inovação social, inovação educacional e inovação nos processos de gestão.

Porém, observou-se uma desmotivação intrínseca em grande parte dos servidores da UFRN, justificada muitas vezes pelo tempo de serviço, atrelados à falta de oportunidades de serem ouvidos ou pela falta de uma cultura voltada para a melhoria contínua; por não haver nenhum 
mecanismo sistematizado capaz de promover a colaboração deles no que diz respeito à proposta de ideias que possam proporcionar a melhoria das atividades da universidade.

A fim de alinhar sua visão estratégica, a Universidade buscar fortalecer uma cultura voltada para a melhoria do trabalho por meio da gestão participativa a partir de iniciativas inovadoras como o Escritório de Ideias e Inovação.

O Escritório de Ideias e Inovação é uma iniciativa conjunta do Departamento de Engenharia de Produção e da PROGESP, com o objetivo de, por meio da inovação aberta, desenvolver o potencial criativo da comunidade universitária e promover uma gestão participativa visando à melhoria dos serviços prestados à comunidade. Seu escopo está voltado a questões relacionadas à Gestão de Pessoas, considerando de forma transversal algumas subáreas da gestão, como: Desenvolvimento Profissional, Qualidade de Vida, Segurança do Trabalho.

\subsection{Focus group}

As contribuições e discussões foram organizadas a partir de questões norteadoras, distinguindo os aspectos positivos, negativos e as oportunidades de melhorias acerca do modelo proposto. Dessa forma, buscou-se validar todos os elementos em estudo, sendo eles: Filtro popular e técnico; Detalhamento das ideias; Avaliação final por multicritérios e análise consensual; e Fatores influenciadores.

\subsection{Elementos para uso da abordagem Crowdsourcing no Escritório de Ideias e Inovação}

\subsubsection{Filtro popular e técnico}

O filtro popular é realizado concomitantemente com a etapa de geração de ideias via abordagem crowd wisdom (HOWE, 2008), sendo realizado pelos próprios idealizadores no momento em que os mesmos participam com "pitacos" e curtidas, conforme abordagem crowd voting (HOWE, 2008). Assim, cada ideia possuirá um Índice de Popularidade (IP), calculado como resultado da fórmula: $\mathrm{IP}=\mathrm{N}^{\mathrm{o}}$ Curtidas $+\mathrm{N}^{\mathrm{o}}$ "pitacos". Ainda nessa fase, $\mathrm{o}$ time de especialistas avaliadores realiza o filtro técnico, cujo objetivo é selecionar ideias que não foram tão populares entre a multidão, mas que possuam potencial para a instituição.

\subsubsection{Detalhamento das ideias}

Com o objetivo de minimizar os fatores que influenciam o processo de avaliação (ver Quadro 4), os especialistas avaliadores acompanharão esse elemento de detalhamento a fim de conhecer mais profundamente as ideias e sanar, durante o processo, todas as dúvidas acerca de suas especificações. Esse elemento será coordenado por membros do Escritório de Ideias e Inovação, e o idealizador e os co-idealizadores, se houver, poderão aperfeiçoar suas ideias.

\subsubsection{Avaliação final por multicritérios e análise consensual}

Encerrando um processo, os avaliadores devem realizar uma avaliação final. A mesma ocorrerá por meio de análise consensual, utilizando multicritérios. Os critérios utilizados no modelo são os sugeridos por Dean et al. (2006), apresentados no Quadro 2: originalidade, aceitabilidade, implementabilidade, aplicabilidade e efetividade.

A análise consensual é válida quando há concordância entre os avaliadores. Aquelas ideias que obtiverem um grau de concordância acima de 0,7 serão consideradas e o resultado final deve ser classificado conforme a média geral obtida pelas ideias (HENNESSEY; AMABILE, 2011).

\subsubsection{Fatores influenciadores}


Compondo um elemento transversal, todo o processo de avaliação de ideias, mais especificamente as pessoas que o compõe, são influenciadas direto e indiretamente por alguns fatores, os quais devem ser estudados e considerados pelas organizações: Ambiguidade das ideias, apresentação das ideias e clareza do problema, condição de trabalho, conhecimento da organização, contex to histórico e cultural, entendimento dos critérios, motivação, ownership, perfil dos avaliadores e quantidade de ideias (Quadro 4).

\section{CONSIDERAÇÕES, LIMITAÇÕES E RECOMENDAÇÕES}

Considerando um ambiente onde as organizações necessitam frequentemente aplicar melhorias e inovação em seus produtos e processos, a open innovation surgiu como uma nova forma de se gerar soluções, utilizando para isso a integração de pessoas externas à organização. Com a evolução dos recursos tecnológicos, várias iniciativas começaram a surgir sob a prática do crowdsourcing, que sistematiza o processo de inovação por meio da rede online, recompensando de alguma forma os interessados em participar com ideias.

Aplicando essa abordagem em uma instituição pública, o Escritório de Ideias e Inovação busca captar ideias da comunidade universitária da UFRN para solucionar problemas estratégicos, empoderando os envolvidos, que além de contribuir irão avaliar as propostas. $\mathrm{O}$ desafio do crowdsourcing será a assertividade na seleção das ideias a serem desenvolvidas.

Para isso, em uma sessão de focus group com representantes de várias áreas da Universidade, foram validados os elementos que devem compor um sistema de avaliação, considerada como um processo e não uma etapa isolada, utilizando ferramentas construtivas e integradoras, como a ferramenta de detalhamento de ideias e a análise consensual.

Como resultado da análise da literatura e da sessão de validação, os elementos apresentados para compor um sistema de avaliação são:

- Filtro popular e técnico - considerando o tipo de crowdsourcing, segundo Howe (2008): crowd wisdom e crowd voting;

- Critérios de avaliação - considerando a análise por multicritérios (Dean et al., 2006) e análise consensual (HENNESSEY; AMABILE, 2011): originalidade, aceitabilidade, implementabilidade, aplicabilidade e efetividade; com índice de concordância de 0,7 ou mais;

- Fatores que influenciam o processo de avaliação - considerando o Quadro 4, com a análise de diversos autores: ambiguidade das ideias, apresentação das ideias e clareza do problema, condição de trabalho, conhecimento da organização, contexto histórico e cultural, entendimento dos critérios, motivação, ownership, perfil dos avaliadores e quantidade de ideias;

- Características da UFRN e do modelo utilizado pelo Escritório de Ideias e Inovação: ferramenta de detalhamento de ideias.

Para estruturar o sistema de avaliação, se faz necessário estudar, de forma mais aprofundada, a relação entre os indicadores - curtidas e "pitacos" - utilizados na avaliação popular, e como a ponderação entre os mesmos influencia na qualidade da avaliação. Ainda, pode-se realizar estudos futuros acerca do comportamento do sistema de avaliação sob a ótica de outras iniciativas crowdsourcing. Para isso, sugere-se realizar pesquisas do tipo pesquisa-ação.

\section{REFERÊNCIAS}

AMABILE, T. M. Componential Theory of Creativity (Working Paper No. 12096). 2012. 
BLAIR, C. S.; MUMFORD, M. D. Errors in idea evaluation: Preference for the unoriginal?.The journal of creative behavior, v. 41, n. 3, p. 197-222, 2007.

BLOHM, I., RIEDL, C., LEIMEISTER, J. M., \& KRCMAR, H.. Idea evaluation mechanisms for collective intelligence in open innovation communities: Do traders outperform raters? In: Proceedings of 32nd International Conference on Information Systems. 2011.

BLOHM, I., RIEDL, C., FÜLLER, J., \& LEIMEISTER, J. M. . et al. Rate or Trade? Identifying Winning Ideas in Open Idea Sourcing. Information Systems Research, v. 27, n. 1, p. 27-48, 2016.

BRABHAM, D. C. Crowdsourcing as a model for problem solving an introduction and cases. Convergence: the international journal of research into new media technologies, v. 14, n. 1, p. 75-90, 2008.

Crowdsourcing. Mit Press, 2013.

CAROFF, X.; BESANÇON, M. Variability of creativity judgments. Learning and individual differences, v. 18 , n. 4, p. 367-371, 2008.

CHIU, C.; LIANG, T.; TURBAN, E. What can crowdsourcing do for decision support?. Decision Support Systems, v. 65, p. 40-49, 2014.

CLUZEL, F. YANNOU, B., MILLET, D., LEROY, Y. Eco-ideation and eco-selection of R\&D projects portfolio in complex systems industries. Journal of Cleaner Production, v. 112, p. 4329-4343, 2016.

CORREA, C.H., FERREIRA D. M., A. Method for decision making in the management of innovation: criteria for the evaluations of ideas. In: Presented at the International Association for Management of Technology, pp. 2151e2169. 2015.

CRESWELL, J. W. Projeto de pesquisa métodos qualitativo, quantitativo e misto. In: Projeto de pesquisa métodos qualitativo, quantitativo e misto. Artmed, 2010.

CSIKSZENTMIHALYI, M. Edited extract from R. Sternberg (Ed)(1999). Handbook of creativity. 1999.

A systems perspective on creativity. London: Sage Publications, 2001.

DEAN, D. L., HENDER, J. M., RODGERS, T. L., \& SANTANEN, E. et al. Identifying quality, novel, and creative ideas: constructs and scales for idea evaluation. Journal of the Association for Information Systems, Vol. 7, pp. 646-698, 2006.

EBNER, W.; LEIMEISTER, J. M.; KRCMAR, H. Community engineering for innovations: the ideas competition as a method to nurture a virtual community for innovations. R\&d Management, v. 39, n. 4, p. 342356, 2009.

ELING, K.; LANGERAK, F.; GRIFFIN, A. The performance effects of combining rationality and intuition in making early new product idea evaluation decisions. Creativity and Innovation Management, v. 24, n. 3, p. 464-477, 2015.

EUBANKS, D. L.; MUMFORD, M. D. Leader errors and the influence on performance: An investigation of differing levels of impact. The Leadership Quarterly, v. 21, n. 5, p. 809-825, 2010.

GABRIEL, A., CAMARGO, M., MONTICOLO, D., BOLY, V., \& BOURGAULT, M. Improving the idea selection process in creative workshops through contextualisation. Journal of Cleaner Production, 2016.

GEIGER, D., ROSEMANN, M., FIELT, E., \& SCHADER, M. et al. Crowdsourcing information systemsdefinition, typology and design. Thirty Third International Conference on Information Systems, Orlando, 2012.

GIL, A. C. Como elaborar projetos de pesquisa. 4. ed. São Paulo: Atlas, 2009.

GRAY, D.; BROWN, S.; MACANUFO, J. Gamestorming: A playbook for innovators, rulebreakers, and changemakers. O'Reilly Media, Inc., 2010.

GUTIÉRREZ, E. Managing Ambiguity When Evaluating and Selecting New Ideas in Project Portfolio Management. International Journal of Innovation and Technology Management, v. 11, n. 05, p. 1450030, 2014.

HAO, N., KU, Y., LIU, M., HU, Y., BODNER, M., GRABNER, R. H., \& FINK, A. Reflection enhances creativity: Beneficial effects of idea evaluation on idea generation. Brain and cognition, v. 103, p. 30-37, 2016.

HENNESSEY, B. A.; AMABILE, T. M. Consensual assessment. Encyclopedia of creativity, v. 1, p. 253-260, 2011. 
HERMAN, A.; REITER-PALMON, Roni. The effect of regulatory focus on idea generation and idea evaluation. Psychology of Aesthetics, Creativity, and the Arts, v. 5, n. 1, p. 13, 2011.

HOWE, J. Crowdsourcing: How the power of the crowd is driving the future of business. Random House, 2008.

JAGPAT, S. et al. Interdependency between average novelty, individual average novelty, and variety. International Journal of Design Creativity and Innovation, v. 3, n. 1, p. 43-60, 2015.

KAHRAMAN, C.; BÜYÜKÖZKAN, G.; ATEŞ, N. Y. A two phase multi-attribute decision-making approach for new product introduction. Information Sciences, v. 177, n. 7, p. 1567-1582, 2007.

KOBAYASHI, M. et al. Collaboration Support System for Analyzing Individual Differences Based on Designers' Idea Evaluation. In: DS 58-5: Proceedings of ICED 09, the 17th International Conference on Engineering Design, Vol. 5, Design Methods and Tools (pt. 1), Palo Alto, CA, USA, 24.-27.08. 2009.

KUDROWITZ, B. M.; WALLACE, D. Assessing the quality of ideas from prolific, early-stage product ideation. Journal of Engineering Design, v. 24, n. 2, p. 120-139, 2013.

LIIKKANEN, L. A. et al. Quantitative evaluation of the effectiveness of idea generation in the wild. In: International Conference on Human Centered Design. Springer Berlin Heidelberg, 2011. p. 120-129.

MAGNUSSON, P. R.; NETZ, J.; WÄSTLUND, E. Exploring holistic intuitive idea screening in the light of formal criteria. Technovation, v. 34, n. 5, p. 315-326, 2014.

ONARHEIM, B.; CHRISTENSEN, B. T. Distributed idea screening in stage-gate development process. Journal of Engineering Design, v. 23, n 9, p 660-673, 2012.

REITER-PALMON, R.; ILLIES, J. J. Leadership and creativity: Understanding leadership from a creative problem-solving perspective. The Leadership Quarterly, v. 15, n. 1, p. 55-77, 2004.

RIEDL, C. et al. The effect of rating scales on decision quality and user attitudes in online innovation communities. International Journal of Electronic Commerce, v. 17, n. 3, p. 7-36, 2013.

RIETZSCHEL, E. F.; NIJSTAD, B. A.; STROEBE, Wolfgang. Productivity is not enough: A comparison of interactive and nominal brainstorming groups on idea generation and selection. Journal of Experimental Social Psychology, v. 42, n. 2, p. 244-251, 2006.

Rio Grande do Norte. Pró-Reitoria de Gestão de Pessoas da UFRN. Planejamento Estratégico. 2016. Disponível em: <www.progesp.ufrn.br〉. Acesso em: 05 set. 2016.

Rio Grande do Norte. Universidade Federal do Rio Grande do Norte. Plano de Desenvolvimento Institucional. 2016. Disponível em: <www.ufrn.br>. Acesso em: 04 set. 2016.

Rio Grande do Norte. Universidade Federal do Rio Grande do Norte. Plano de Gestão. 2016. Disponível em: <www.ufrn.br>. Acesso em: 04 set. 2016.

SAXTON, G. D.; OH, O.; KISHORE, R. Rules of crowdsourcing: Models, issues, and systems of control. Information Systems Management, v. 30, n. 1, p. 2-20, 2013.

SOARES, A. V. A. Inovação no setor público: obstáculos e alternativas. 2009. Disponível em: www.administradores.com.br/. Acesso em 02. Jul 2015.

TUNG, W.; YUAN, S.; TSAI, J. A custom collaboration service system for idea management of mobile phone design. Human Factors and Ergonomics in Manufacturing \& Service Industries, v. 19, n. 5, p. 494-509, 2009.

VERHAEGEN, P.; VANDEVENNE, D; PEETERS, J.; DUFLOU, J. Refinements to the variety metric for idea evaluation. Design Studies, v. 34, n. 2, p. 243-263, 2012.

VON OECH, R. Um chute na rotina: os quatro papéis essenciais do processo criativo. São Paulo. Cultura editores associados, 1994.

XIE, L.; ZHANG, P. Idea Management System for Team Creation. Journal of software, v. 5, n. 11, p. 1187 $1194,2010$.

YANG, Y. Open innovation contests in online markets: idea generation and idea evaluation with collective intelligence. Temple University Graduate Board. 2012.

YIN, R. K. Estudo de Caso: Planejamento e Métodos. Bookman editora, 4. ed. Porto alegre: bookman. 2010. 